Radial and Nonradial Pulsations as Probes of Stellar Physics

ASP Conference Series, Vol. 259, 2002

C. Aerts, T.R. Bedding, \& J. Christensen-Dalsgaard, eds.

\title{
Spectroscopic Monitoring of 10 Northern SPB Candidates ${ }^{1}$
}

\author{
P. Mathias, J.-M. Le Contel
}

Observatoire de la Côte d'Azur, UMR 6528, Nice, France

C. Aerts, P. De Cat, H. Van Winckel, E. Robbrecht

Instituut voor Sterrenkunde, Katholieke Universiteit Leuven, Belgium

\section{Briquet}

Institut d'Astrophysique et de Géophysique, Liège, Belgium

\section{J. Cuypers}

Koninklijke Sterrenwacht van België, Brussel, Belgium

\begin{abstract}
We present the results of 1.5 years of spectroscopic monitoring of 10 bright northern SPB candidates discovered from the Hipparcos mission. All stars have line-profile variations and are periodic, with periods corresponding to high-order $g$-modes. We therefore confirm them to be new SPBs.
\end{abstract}

\section{Introduction}

Because of the many awaited space missions, the coming decade will be important for asteroseismology. In order to study the deep internal layers, $g$-modes pulsators, such as Slowly Pulsating B stars (hereafter SPBs), are the best objects. The HIPPARCOS mission led to the discovery of many new SPBs candidates (Waelkens et al., 1998). However, some of these candidates turned out not to be SPBs (see De Cat, 2001), and spectroscopic data are needed to confirm the pulsational nature of the targets.

A total of 10 objects was selected from the list provided by Waelkens et al. (1998). Note that HD 147394 is discussed separately (Briquet et al., these proceedings). Depending on the brightness of each star, between 15 and 60 spectra were obtained, with a signal-to-noise ratio around 100 and a resolving power of 15000 in the Si II $\lambda \lambda 4128,4130$ region.

\footnotetext{
${ }^{1}$ The follow-up data were obtained with the $1.52 \mathrm{~m}$ telescope of the Haute-Provence Observatory in France
} 


\section{Results}

The frequency analysis was performed both on HIPPARCOS (search for multiperiodicity) and radial velocity data (validation of the Hipparcos frequency). Results are provided in Table 1.

Table 1. Pulsation characteristics of the SPBs. The $f_{p}$ and $f_{s}$ values, respectively, correspond to the dominant photometric and spectroscopic frequencies. The pulsation constant $Q_{p}$ is computed from the $f_{p}$ value. No value $f_{s}$ is provided for HD 140873 because it is a doublelined spectroscopic binary and the companion could not be removed.

\begin{tabular}{rllc}
\hline Star $(\mathrm{HD})$ & $f_{p}\left[\mathrm{c} \mathrm{d}^{-1}\right]$ & $f_{s}\left[\mathrm{~cd}^{-1}\right]$ & $Q_{p}\left[\mathrm{c} \mathrm{d}^{-1}\right]$ \\
\hline 1976 & 0.93914 & 0.39934 & 0.38 \\
21071 & 1.18843 & 1.18843 & 0.52 \\
25558 & 0.65284 & 0.65284 & 0.69 \\
28114 & 0.79104 & 0.79104 & 0.42 \\
138764 & 0.794 & 0.794 & 0.62 \\
140873 & 1.149 & - & 0.55 \\
182255 & 0.7922 & 0.7922 & 0.67 \\
206540 & 0.7624 & 0.7624 & 0.54 \\
208057 & 0.80172 & 0.89045 & 0.48 \\
\hline
\end{tabular}

All target stars show line profile variations on time scales expected for non-radial $g$-mode pulsations. All but one, HD 28114, show evidence for multiperiodicity. The latter star still should be considered as an SPB star since line profiles are well-marked, the radial velocity curve is sinusoidal and the star presents "normal" abundances (Adelman \& Philip, 1996). See also Briquet et al. (these proceedings).

Two stars (HD 1976 and HD 208057) present different dominant frequencies in spectroscopy and photometry: this could indicate different modes of high and low degree, respectively, but both stars are also rapid rotators and possibly members of binary systems.

\section{References}

Adelman, S.J. \& Philip, A.G.D. 1996, MNRAS, 282, 1181

De Cat, P. 2001, PhD Thesis, University of Leuven, Belgium

Waelkens, C., Aerts, C., Kestens, E., Grenon, M., \& Eyer, L. 1998, A\&A, 330, 215 